\title{
Weak and Nondiscriminative Responses to Conspecifics in the Rat Hippocampus
}

\author{
Moritz von Heimendahl, Rajnish P. Rao, and Michael Brecht \\ Bernstein Center for Computational Neuroscience, Humboldt University of Berlin, 10115 Berlin, Germany
}

Little is known about how hippocampal neurons in rodents respond to and represent conspecifics. To address this question, we let rats interact while quantifying hippocampal neuronal activation patterns with extracellular recordings and immediate-early gene (c-Fos) expression. A total of 319 single putative pyramidal neurons was recorded in dorsal hippocampus. In sessions with multiple stimulus rats, no cell responded differentially to individual rats $(N=267$ cells $)$. We did find, however, that the presence of other rats induced a significant enhancement or suppression of firing in a fraction of neurons $(n=22$ of $319 ; 7 \%)$. As expected, a large fraction of neurons ( $n=$ $170 ; 53 \%)$ had place fields. There was no evidence for place-independent responses to rats. Rather, the modulations were linked to the spatial responses. While neurons did not discriminate between individual rats, they did discriminate between rats and inanimate objects. Surprisingly, neuronal responses were more strongly modulated by objects than by rats, even though subjects spent more time near their conspecifics. Consistent with the low fraction of rat-modulated cells, social encounters did not induce c-Fos expression in the hippocampus, while there was a social interaction-specific expression in the basolateral amygdala. In both interacting and non-interacting rats, the fraction of c-Fos-expressing cells in the hippocampus was very low. Our investigation of social coding in the rat hippocampus, along with other recent work, showed that social responses were rare and lacked individual specificity, altogether speaking against a role of rodent dorsal hippocampus in social memory.

\section{Introduction}

Rodents form complex social memories. They can discriminate between conspecifics (Husted and McKenna, 1966; Petrulis, 2009) and memorize them (Thor and Holloway, 1982). Moreover, they can match individual animals to their odors (Gheusi et al., 1997) or even one of an individual's multiple olfactory features to another (Johnston and Jernigan, 1994). This suggests an integrated representation of conspecifics, and therefore the hippocampus, which receives integrated input from most if not all sensory cortices (Burwell, 2000), has long been a candidate in the search for a neural correlate of social memory.

The hippocampus plays a key role in learning and memory (Scoville and Milner, 1957; O'Keefe and Nadel, 1978; Squire, 1992). Social memories, among others, are dramatically impaired upon lesion of the temporal lobe (Scoville and Milner, 1957), even when limited to the CA1 subfield of the hippocampus (ZolaMorgan et al., 1986). The idea that the human hippocampus

\footnotetext{
Received July 26, 2011; revised Nov. 23, 2011; accepted Dec. 4, 2011

Author contributions: M.v.H., R.P.R., and M.B. designed research; M.v.H. and R.P.R. performed research; M.v.H. and R.P.R. analyzed data; M.v.H., R.P.R., and M.B. wrote the paper.

This work was supported by Humboldt Universität zu Berlin, the Bernstein Center for Computational Neuroscience Berlin, the German Federal Ministry of Education and Research (Bundesministerium für Bildung und Forschung) (Förderkennzeichen 01GQ1001A), Neurocure, and a European Research Council grant (M.B.). We thankGabi Ziermann, Brigitte Geue, and Undine Schneeweiss for technical assistance. Jason H. Wolfe, Alison Barth, Andrea Burgalossi, Edith Chorev, John Tukker, and Claudia Roth-Alpermann gave valuable comments on this manuscript. The authors declare no competing financial interests.

Correspondence should be addressed to either Moritz von Heimendahl or Michael Brecht, Bernstein Center for Computational Neuroscience, Humboldt University of Berlin, Philippstrasse 13 Haus 6, 10115 Berlin, Germany. E-mail: moritz.vonheimendahl@bccn-berlin.de or michael.brecht@bccn-berlin.de.

DOI:10.1523/JNEUROSCI.3812-11.2012

Copyright $\odot 2012$ the authors $\quad 0270-6474 / 12 / 322129-13 \$ 15.00 / 0$
}

represents information related to recognition memory of conspecifics is supported by recent data from neuronal recordings in humans, in which responses to individual persons (Quian Quiroga et al., 2005) have been found.

While the primate hippocampus has long been attributed a broad role in memory, in the rodent many studies focused on the role of the hippocampus in spatial navigation and memory (O'Keefe and Nadel, 1978). Its involvement in nonspatial memory has been shown (Eichenbaum et al., 1999), but the extent and importance of this role are debated (Eichenbaum et al., 1999; O’Keefe, 1999; Redish, 2001).

Lesion studies have led to conflicting results about the role of rodent hippocampus in social memory formation. Most (Bannerman et al., 2001; Petrulis and Eichenbaum, 2003; Squires et al., 2006; Feinberg et al., 2012) but not all (Kogan et al., 2000; Uekita and Okanoya, 2011) studies have shown that rodents do not need the hippocampus to recognize conspecifics as familiar. A study in hamsters suggested that so-called recollection memory does require an intact hippocampus: when dorsal CA1 was reversibly inactivated during an encounter with familiar individuals, the subject failed to associate the respective memories (Lai et al., 2005). Also, socially transmitted food preference seems to rely partly on the hippocampus (Winocur, 1990).

To summarize, in humans there is electrophysiological evidence for hippocampal social representations, and in rodents, lesion studies suggest its involvement in social interactions, but direct electrophysiological evidence is missing. We therefore used a combination of electrophysiology and immunohistochemistry to ask the following questions: (1) Are there hippocampal responses to conspecifics (i.e., rat cells)? (2) Are there individual- 
specific cells? (3) Are the rat neurons, if any, independent of context, or do they show so-called conjunctive responses (i.e., respond to a rat in a given place)? (4) What is the anatomical distribution of socially modulated neurons?

\section{Materials and Methods}

\section{Electrophysiology experiments}

Animals. Experimental animals comprised subject rats and stimulus rats. Both were Wistar rats, weighing 130-370 g, from Harlan. They were housed in groups of two to three until surgery, after which protection of the implant made individual housing necessary. The light cycle was inverted for most experiments so that the behavioral sessions took place during the dark phase.

In electrophysiology experiments, rats were given $15 \mathrm{~g}$ of rat chow a day and water ad libitum. On days with experimental sessions, subject rats were given food only afterward.

Subject rats were male, while stimulus animals included both male and female rats, former cage mates, and strangers.

All experiments complied with German regulations on animal welfare and were approved by an ethics committee.

Surgery. For electrophysiological experiments, subject rats were implanted with a chronic multielectrode microdrive (Harlan 8-drive; Neuralynx) with eight tetrodes in one or two bundles or in a single linear row. A craniotomy and durectomy were made at variable coordinates, between $1.8-5 \mathrm{~mm}$ caudal and $0-3 \mathrm{~mm}$ lateral of bregma over the right hemisphere. The microdrive was fixed with dental cement after the exposed brain was covered with biocompatible silicon (KwikSil; World Precision Instruments). The microdrive implant allowed mounting of two colored LEDs that were used to track head position during recording sessions.

Animals had unlimited access to food and water postsurgery. Recording sessions began after a $7 \mathrm{~d}$ recovery period.

Behavior. The apparatus (see Fig. $1 A$ ) consisted of a central elevated platform $(45 \times 15 \mathrm{~cm})$ for the subject rat, and two small platforms $(15 \times$ $20 \mathrm{~cm}$ ) for the stimulus rats, separated by a gap of $15 \mathrm{~cm}$. The stimulus rat platforms had walls (height, $20 \mathrm{~cm}$ ) on all sides except the one facing the central platform. All experiments were performed in the dark. Infrared light, invisible to rats, was used as illumination for videography.

Subject and stimulus rats had separate acclimatization sessions to accustom the animals to the new environment. The subject's habituation sessions took place before surgery.

For recording sessions, the subject rat was placed on the central platform. Brain activity and head position were recorded simultaneously. There was no explicit task for the rat to perform except to sample the environment. When necessary, pieces of rat food were manually thrown on the central platform in random locations to encourage locomotion.

Every session consisted of multiple trials. During some of these, stimulus rats were placed on the small platforms. Thus, there were trials with no stimulus rats present, with a stimulus rat on one or both of the platforms, and with their positions inverted. The total duration of a session varied between 584 and $3424 \mathrm{~s}$, and the number of trials (i.e., of switches of the stimulus rat arrangement) ranged from 3 to 69 , with at least 10 switches for more than one-half of the recorded neurons. Each trial could contain multiple sampling episodes. More than the durations of trials and sessions, what mattered for the present study were the times when subject rats approached the stimulus platforms and interacted with stimulus rats, if present. This was not under the experimenter's control. Therefore a post hoc data inclusion criterion was used, excluding data for which the cumulative time for a given condition and location was below a threshold (see below, Rat modulation index).

For a subset of sessions, inanimate objects were placed on the stimulus rat platforms during some trials. These objects varied from session to session and were random items from the laboratory (e.g., an Eppendorf rack filled with paper towels, an empty glove box with a cleaning cloth on it, etc.).

Each subject rat performed between 5 and 17 sessions. For six subject rats, the stimulus rats were always the same two to three rats; one subject rat performed 5 sessions with one pair of stimulus rats and then switched to a new pair for another 12 sessions.
Recording sessions depended on the presence of neurons. Often, they took place on consecutive days; sometimes they were separated by several days. Only once, two recording sessions took place during the same day ( $>7 \mathrm{~h}$ apart).

Electrophysiology. Multiple single-neuron activity was collected with eight independently movable tetrodes made of $25-\mu \mathrm{m}$-diameter platinum/iridium wire, coated with polyimide (California Fine Wire Company). Electrodes were lowered to the pyramidal cell layer of the dorsal hippocampus. Recording sessions began when high-quality unit activity could be recorded. Between sessions, electrodes were advanced by, typically, $40 \mu \mathrm{m}$, and the tissue was allowed to relax overnight. If there was unit activity on at least one tetrode, a recording session took place; otherwise, electrodes were further advanced. Some electrodes were recorded from in several different hippocampal regions, as for example CA1 and then, deeper along the same track, CA3 or dentate gyrus (DG). Electrode depth was estimated from drive turn count, refined by observation of hippocampus-typical complex spikes and theta-modulated firing.

After passing through a unity-gain headstage (Neuralynx), signals were transmitted through a tether cable to a programmable amplifier (Digital Lynx; Neuralynx). The spike signals were amplified by a factor of 10 and then digitized at $32 \mathrm{kHz}$. The digital signal was bandpass filtered between $600 \mathrm{~Hz}$ and $6 \mathrm{kHz}$. Events that reached a user-set threshold were recorded for $1 \mathrm{~ms}$ ( $250 \mu$ s before voltage peak and $750 \mu$ s after peak).

Histology. After the last recording session, rats were deeply anesthetized with ketamine and xylazine and perfused transcardially. Brains were fixed in $4 \%$ paraformaldehyde, cut in $150-\mu \mathrm{m}$-thick coronal sections, and stained for cytochrome oxidase or with biocytin. Recording locations were determined as the intersection of the electrode tracks with the pyramidal (or granule) cell layer or by electric lesions. For some rats, the individual electrodes within a bundle could not be discerned and an average position across the electrodes was used.

Locations were classified as fasciola cinereum (FC), CA1, CA2, lateral CA3, or CA3/DG. Rostrocaudal positions were inferred by matching landmarks in the sections and the rat atlas (Paxinos and Watson, 2007).

To determine the proximodistal position of recording sites within CA1, the distance from the CA2-CA1 transition to the electrode location was divided by the full length of the pyramidal cell layer of CA1.

Spike sorting. Spikes were sorted off-line on the basis of their amplitude and principal components by means of a semiautomatic clustering algorithm (KlustaKwik; 2000; written by K. D. Harris, Rutgers University, Newark, NJ). The resulting classification was corrected and refined manually with MClust software (written by A. D. Redish, University of Minnesota, Minneapolis, MN) running in MATLAB (MathWorks). Between 0 and 20 (median, 3) single units were isolated from each tetrode, based on refractory period, stability, and separation quality. Refractoriness was quantified by counting the number of interspike intervals (ISIs) that were $<2 \mathrm{~ms}$, normalized by the number of ISIs $<10$ $\mathrm{ms}$. Units were considered single neurons if this ratio was $<0.05$. Separation quality was assessed using L-ratio and isolation distance (Schmitzer-Torbert et al., 2005). As in the study by Schmitzer-Torbert et al. (2005), clusters were considered well separated with an L-ratio $<0.2$ and isolation distance $>15$. An extended dataset of less well separated neurons was defined by L-ratio $<0.5$, no restriction on isolation distance, and the same ISI criterion as above. Neurons firing at an average rate of $>5 \mathrm{~Hz}$ were considered interneurons, and otherwise pyramidal neurons.

Videography. The setup was filmed with two cameras. A color camera was used to track the LEDs on the animal's head. Positions were extracted by Digital Lynx (Neuralynx) and custom-written MATLAB code. Moreover, the setup was lit with near-infrared light and filmed with a monochrome camera at 15 or 30 frames per second. These films were used for qualitative analysis of rat behavior (e.g., to exclude times when the subject rat was grooming). For a subset of recording sessions, times of snoutto-snout interactions were extracted manually.

Firing maps. To relate head location to firing rate, color-coded firing maps were plotted. For these, space was discretized into pixels of $2.5 \times$ 
$2.5 \mathrm{~cm}$, for which the occupancy $z$ of a given pixel $x$ was calculated as follows:

$$
z(x)=\sum_{t} w\left(x-x_{t}\right) \Delta t
$$

where $x_{t}$ is the position of the rat at time $t, \Delta t$ is the interframe interval, and $w$ is a Gaussian smoothing kernel with $\sigma=5 \mathrm{~cm}$ [adapted from the study by Harris et al. (2001)].

Then, the firing rate $r$ was calculated as follows:

$$
r(x)=\frac{\sum_{i} w\left(x-x_{i}\right)}{z},
$$

where $x_{i}$ is the position of the rat when spike $i$ was fired. The firing rate of pixels whose occupancy $z$ was $<100 \mathrm{~ms}$ was considered unreliable and not shown.

Occupancy and firing maps were calculated for each half of the maze separately (indicated in figures by a dashed line) and averaged across trials with the same stimulus rat, object, or nothing in the respective gap.

For the relative occupancy map, with-rat and without-rat episodes were analyzed separately as follows: If there was a stimulus rat on the small platform in the half of the maze where the subject rat was, this was considered a with-rat episode, and otherwise, a without-rat episode. In particular, this means that trials without any stimulus rats contributed only without-rat episodes, no matter in which half the subject rat was, while trials with two stimulus rats contributed only with-rat episodes. All with-rat episodes were summed across trials, sessions, and both halves of the maze. The occupancy was calculated using the above formula for $z$ and normalized by the total time spent in the respective maze half.

Reach index. To quantify how far the subject rat reached out into the gap between the platforms when there was or was not a stimulus rat present, a reach index was calculated. It ranged from 0 to 1 for head positions on the near and the far side, respectively. For each session, the average reach index was calculated as an average across all episodes during which the subject rat's head was in the gap, separately for episodes with and without a stimulus rat.

Rat modulation index. Firing rate modulation by the presence of stimulus rats was quantified by comparing the firing rate while the subject rat explored the gap (see Fig. $1 A$, pink shaded area) with and without a stimulus rat. For each neuron, a rat modulation index (RMI) was defined as follows: RMI $=(r-n) /(r+n)$, where $r$ and $n$ are the firing rates with and without a stimulus rat, respectively. $r$ and $n$ were calculated as an average across the trials of a session and across the two gaps. For each gap, trials were weighted by the time the subject rat spent in the gap, while the two gap locations were given equal weight. If the occupancy of a gap in a given condition was $<1 \mathrm{~s}$, the data were discarded.

A bias could have been introduced if rats had a tendency to, for example, lean out further into the gap when there was stimulus rat. In this case, the with-rat and without-rat firing rates would be sampled in two slightly different places. To avoid this, the gap was divided into five zones from near to far from the main platform. The comparison between with-rat and without-rat episodes was done separately by zone, and averaged afterward.

To test whether rat presence significantly modulated firing rates, a permutation test was performed. For this, the labels demarking epochs with rat and without were shuffled and from these data a "fake RMI" was calculated 1000 times. The shuffling was performed by cyclically shifting the labels by a random amount of time with respect to the behavior and spike data (Skaggs et al., 1993). The cyclical shift (instead of random shuffling) preserves the time course of random fluctuations in firing rate. This ensures, in particular, that a drift in firing rate is not mistaken for a coding effect, and more generally gives more statistical power to recording sessions with numerous switches in stimulus configuration. Neurons recorded in the same session were shuffled jointly to preserve noise correlation (Montemurro et al., 2007).

Values of $p$ were obtained by ranking the real RMI in the distribution of fake RMIs.

Place modulation index. A place modulation index (PMI) was defined, designed to be symmetrical in definition to the rat modulation index, as follows: $\mathrm{PMI}=\left(p_{1}-p_{2}\right) /\left(p_{1}+p_{2}\right)$, where $p_{1}$ and $p_{2}$ are the firing rates in one and the other gap. They were calculated across trials with and without stimulus rats separately and then averaged. Trials were weighted by the time the subject rat spent in the gap.

Significance was tested as for the RMI test, by shuffling the two platforms.

Individual modulation index. In a similar way to the RMI, an individual modulation index (IMI) was defined to determine whether neurons fired differently for the presence of one stimulus rat as opposed to another. It was calculated as follows: IMI $=\left(r_{1}-r_{2}\right) /\left(r_{1}+r_{2}\right)$, for all sessions that had two stimulus rats. $r_{1}$ and $r_{2}$ were the average firing rates when the subject rat was near one or the other stimulus rat. Significance was tested as above, using a one-tailed test on |IMI|.

Other indices. Rat-versus-object index, object-modulation index, and interaction-rat modulation index were defined in an analogous way.

Place cell criteria. While the place modulation index above quantified spatial discrimination between the two gaps, for a more general measure of place coding, widely used criteria (Muller et al., 1987) were used: Firing maps were calculated as described above. All pixels with $>30 \%$ of the peak firing rate (of any pixel) were candidates for place fields. These pixels had to form contiguous patches of $>200 \mathrm{~cm}^{2}$, but jointly cover $<90 \%$ of the total sampled area. To determine whether place fields were robust against the presence of stimulus rat, the stability of the firing map across trials was estimated as follows: The session was divided in two parts by choosing a contiguous part of one-half of the length of the session at a random time within the session. The two remaining bits before and after the selected part were the second part. Pearson's correlation coefficient between the two maps of the half-sessions was determined. This procedure was repeated for 100 random divisions. The average correlation coefficient was called the consistency of the neuron.

Spatial information rate. As an alternative measure of spatial modulation, the rate of mutual information between firing rate and the subject rat's location was used (Skaggs et al., 1993). The information rate was calculated as follows:

$$
I=\int \mathrm{d} x r(x) \log _{2} \frac{r(x)}{\bar{r}} z(x) / T,
$$

where $r$ and $z$ are the occupancy and the firing rate of a given pixel as defined above. $\bar{r}$ is the average firing rate, and $T$ is the duration of the session. To subtract bias due to finite data size, shuffled information rates were calculated by cyclically time-shifting the spike train with respect to the subject rat's position. The average of 1000 shuffled values was subtracted from the nonshuffled value for an unbiased estimate of the real information rate. At the same time, ranking of the real information rate in the distribution of the information rates calculated on shuffled data produced the corresponding $p$ value.

\section{Immunohistochemistry experiments}

Animals. Male Wistar rats (250-300 g at the time of experiment) were procured from Harlan when they were 2 weeks of age. They were group housed for 2 weeks with the dam and then subjected to social isolation (individual housing) for a further period of 3 weeks in a temperaturecontrolled room with a $12 \mathrm{~h}$ light/dark cycle and ad libitum access to food and water. Twelve animals from two different litters were used and were randomly assigned to either social interaction or control groups.

All experiments complied with German regulations on animal welfare and were approved by an ethics committee.

Behavior. All behavioral trials were conducted during the dark phase of the light/dark cycle in a dark room with infrared illumination. Following social isolation, all animals were subjected to habituation (10 min each for $5 \mathrm{~d})$ on the behavioral setup that consisted of two platforms $(24 \times 16$ $\mathrm{cm}$, surrounded by $40 \mathrm{~cm}$ walls on three sides) separated by a $15 \mathrm{~cm}$ gap along the short edge, across which the rats could interact. On the day of the experiment, animals in the social interaction group interacted with a non-littermate placed across the gap for $10 \mathrm{~min}$ while the control animals were reexposed to the behavioral setup singly. Following the behavioral trials, the animals were returned to their home cages in the holding room. 
The setup was cleaned with $20 \%$ ethanol between animals and dried with paper towels.

Histochemistry. Animals were deeply anesthetized with $20 \%$ urethane (Sigma-Aldrich) 60-90 min after behavioral trials and perfused transcardically with ice-cold $0.1 \mathrm{M}$ PBS, $\mathrm{pH}$ 7.4, followed by $4 \%$ paraformaldehyde (PFA) in $0.1 \mathrm{M}$ PBS. The brains were dissected out and subjected to postfixation in PFA overnight and then stored in a cryoprotectant ( $20 \%$ glycerol in $0.1 \mathrm{M}$ PBS) until sectioning. Coronal sections ( $40 \mu \mathrm{m}$ thick) cut on a cryostat (Frigomobil; Leica Microsystems) at $-25^{\circ} \mathrm{C}$ were collected in $0.1 \mathrm{~m}$ PBS. While one in four sections were used for freefloating c-Fos immunohistochemistry, an adjacent series of sections was Nissl stained for neuroanatomical comparison. For immunohistochemistry, the sections were washed in $0.1 \mathrm{M}$ PBS (three times; $10 \mathrm{~min}$ each), permeabilized with $3 \% \mathrm{H}_{2} \mathrm{O}_{2}$ in methanol, washed, and blocked for $1 \mathrm{~h}$ at room temperature with blocking solution consisting of $0.1 \mathrm{~m}$ PBS, 1\% BSA (Sigma-Aldrich), and $0.1 \%$ Triton X-100 (Merck). The sections were incubated with fresh blocking solution containing rabbit anti-c-Fos antibody (Ab-5; Calbiochem; Merck; 1:10,000) for $16 \mathrm{~h}$ at room temperature and washed. They were subsequently processed for the avidinbiotin procedure using the Vectastain $\mathrm{ABC}$ Elite peroxidase rabbit IgG kit (Vector Laboratories) with one modification (1:2000 dilution of secondary antibody). Sections were then subjected to 3,3'-diaminobenzidine reaction for 5 min using peroxidase substrate kit DAB (Vector Laboratories) with nickel ions to obtain a dark colored precipitate. The color reaction was stopped with water, sections washed extensively with $0.1 \mathrm{M}$ PBS, and mounted on chrome-alum/gelatin-coated slides. After air drying, they were coverslipped with Mowiol 4-88. For Nissl staining, sections were first mounted on slides and air dried, dehydrated in ethanol grades, stained with $1 \%$ cresyl violet (Sigma-Aldrich), cleared with isopropanol/xylene, and mounted in DPX. All chemicals were from Roth unless otherwise indicated.

Quantification of cell numbers. All brains were coded before sectioning and quantification of c-Fos-positive cells was performed by an experimenter who was blind to the treatment conditions. Mounted sections were viewed using a light microscope (BX51; Olympus) connected to an automated stage controller (MAC 5000; LEP). The nomenclature and boundaries of the various brain areas are as described in the atlas of Paxinos and Watson (2007) except for the fasciola cinereum (Henriksen et al., 2010). The bregma coordinates used for dorsal hippocampus were between -3.0 and $-4.08 \mathrm{~mm}$, for ventral from -4.80 to $-5.28 \mathrm{~mm}$, and for the basolateral amygdala (BLA) sections, the coordinates spanned -2.28 and $-3.48 \mathrm{~mm}$. Clearly identifiable c-Fos-positive nuclei were counted on both left and right hemispheres of five or six sections per animal using unbiased stereology software (Stereoinvestigator; MBF Bioscience) and normalized to the area in which the cell counts were estimated. Furthermore, the exact locations of the positive nuclei in the hippocampus were mapped using the Neurolucida software (Stereoinvestigator; MBF Bioscience) and the $x-y$ coordinates were analyzed with custom-written software (MATLAB; MathWorks).

The total number of CA1 pyramidal neurons in the cell body layer of dorsal hippocampus was similarly estimated using the Nissl-stained sections (four animals).
B
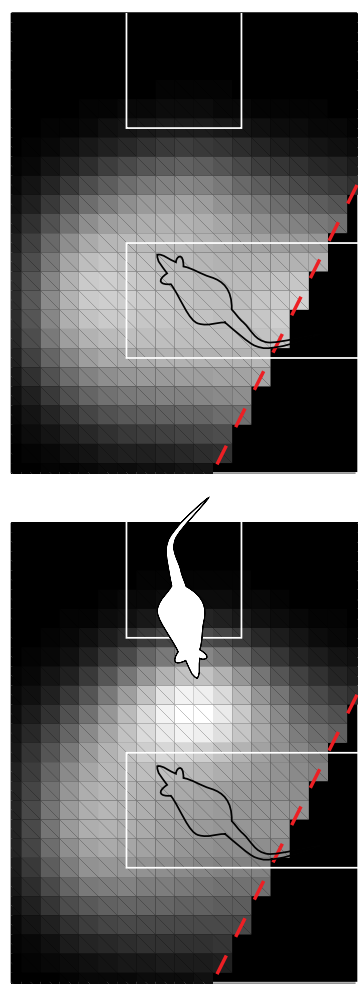

列

0

Reach index without rat

Figure 1. Setup and behavior. $\boldsymbol{A}$, Schematic view of the electrophysiology setup. Elevated platforms are shown in gray and are 列 Relative occupancy of the maze without (top) and with (bottom) a stimulus rat. The lighter shading corresponds to more time spent in a place. Both halves of the maze are shown collapsed across the dashed line (see Materials and Methods). Note how, when there is a stimulus rat, the subject rat's relative occupancy is increased in the gap. $\boldsymbol{C}$, Reach index, quantifying how far the subject rat reached out into the gap, for episodes with versus without a stimulus rat. Each dot is a recording session.

Table 1. Population statistics

\begin{tabular}{lllllll}
\hline Statistic & $\begin{array}{l}\text { Standard } \\
\text { dataset }\end{array}$ & $N$ & $\begin{array}{l}\text { Extended } \\
\text { dataset }\end{array}$ & $N$ & Conservative dataset & $N$ \\
\hline RMI & $0.26^{* * *}$ & 318 & $0.23^{* * *}$ & 454 & $0.24^{* * *}$ & 113 \\
IMI & $0.25^{\text {n.s. }}$ & 267 & $0.22^{\text {n.s. }}$ & 392 & $0.24^{\text {n.s. }}$ & 86 \\
ROI & $0.34^{* * *}$ & 115 & $0.30^{* * *}$ & 215 & $0.30^{* * *}$ & 25 \\
OMl & $0.34^{* * *}$ & 113 & $0.30^{* * *}$ & 213 & $0.31^{* *}$ & 24 \\
\hline
\end{tabular}

Population statistics calculated on different datasets: The standard dataset contains all well separated, single, putative pyramidal neurons. The extended dataset contains the standard dataset plus less well separated neurons (see Materials and Methods for criteria applied). The conservative dataset is a subset of the standard dataset that avoids double sampling due to overlap in recording sites and is a lower bound on the true number of distinct neurons that were recorded from.

Significance is indicated as follows: ${ }^{* *} p<0.01 ;{ }^{* * *} p<0.001$; n.s., not significant.

Analysis of c-Fos cell density. To find out whether there was an effect of treatment on c-Fos expression, cell densities were compared between animals of the social interaction group and the control group. To test significance, a permutation test was used that exchanged group affiliation of rats. For this, in each pair of animals (treatment and control), the difference in cell density was divided by their sum. An exact permutation test was used to test the hypothesis that interaction $>$ control (one-tailed test). 
Table 2. Location of recorded cells

\begin{tabular}{lc}
\hline Subregion of dorsal hippocampal & No. of cells \\
\hline CA1 & 165 \\
Near CA2 & 96 \\
CA3 & 30 \\
CA3/DG & 15 \\
Fasciola cinereum & 13 \\
Total & 319
\end{tabular}

The criteria applied were conservative about attributing cells to CA1. For example, the "near CA2" class may contain some $C A 1$ cells but not vice versa. $C A 3 / D G$ refers to cells from either $D G$ or the part of $C A 3$ that lies between the leaves of the $D G$, while the " $C A 3$ " cells are from the lateral curve of $C A 3$.

A

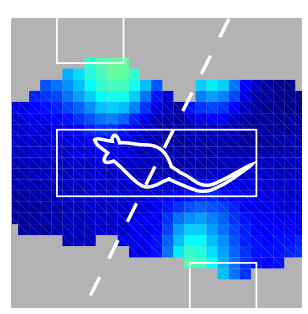

Max Rate 8.1

B

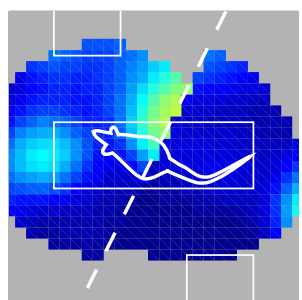

Max Rate 2.9

C

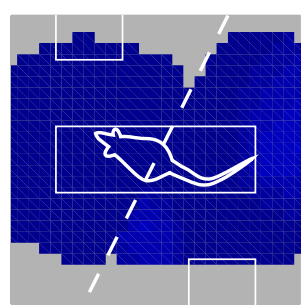

Max Rate 5.9

D

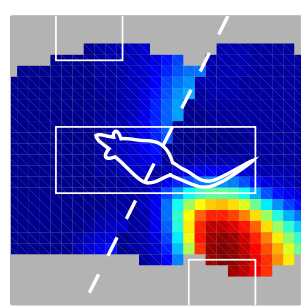

Max Rate 7.5
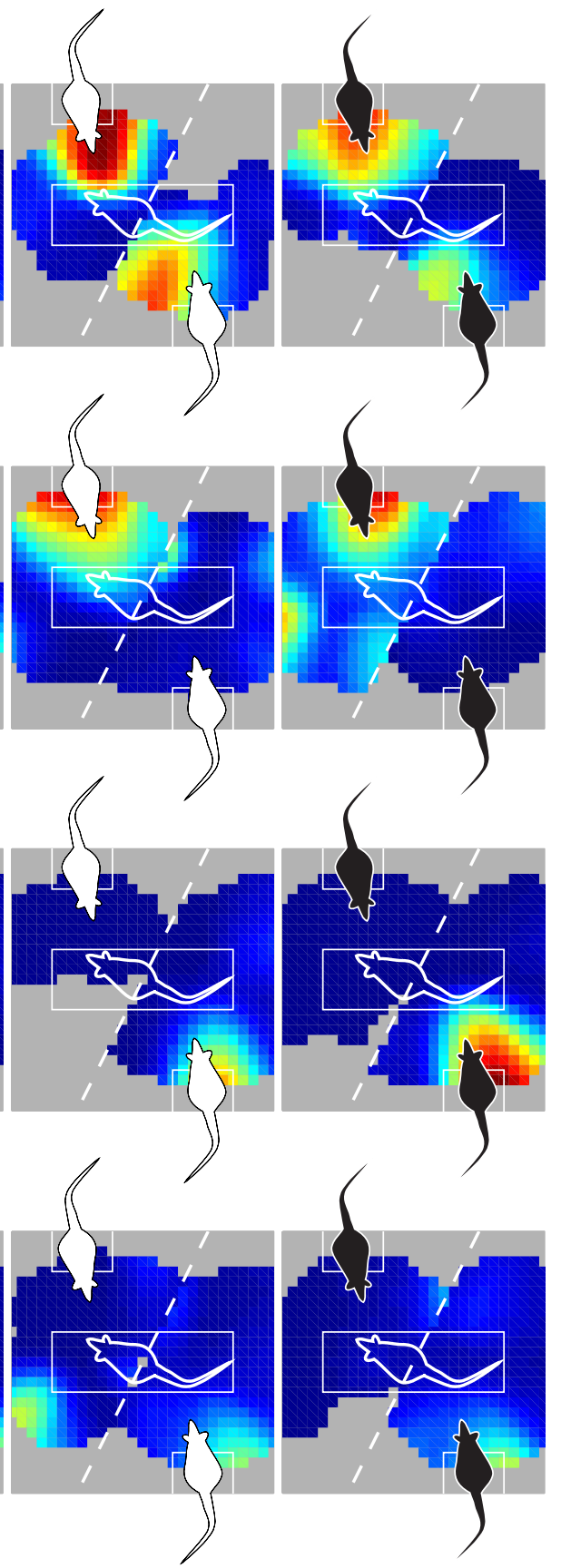

Figure 2. Rat-modulated cells. Firing maps of cells that respond to stimulus rat presence. Deep red is maximal firing rate, and deep blue represents no firing. The subject rat is shown as an outline; stimulus rats are in black or white. $\boldsymbol{A}$, Firing map of a rat-modulated cell. Each subpanel shows data averaged across trials with the same stimulus rat (or none) in the respective gap. For example, the part to the right of the dashed line in the left panel shows data from trials in which there was no stimulus rat on the lower platform. The dashed line indicates that these were not necessarily the same trials represented in the other half of the maze. $\boldsymbol{B}-\boldsymbol{D}$, As in $\boldsymbol{A}$ for other rat-modulated cells. Note that while cell $\boldsymbol{A}$ shows a modulation in both gaps, cells $\boldsymbol{B}-\boldsymbol{D}$ react only in one gap. Unlike cells $\boldsymbol{A}-\boldsymbol{C}$, cell $\boldsymbol{D}$ shows strongly reduced rather than enhanced firing during stimulus rat presence.
In the electrophysiology paradigm, rats were implanted with a chronic electrode drive and spiking activity of cells in the dorsal hippocampus was recorded extracellularly. Experimental sessions were performed in a custom-built maze (Fig. $1 A$ ); the implanted rat ("subject rat") could move freely on a rectangular, elevated platform. "Stimulus rats" were constrained to two small platforms, located at either end of the main platform and separated from it by a gap, allowing whisker and snout contact when both animals extended their heads into the gap. From one trial to another, stimulus rats were added, removed, or their positions switched. As detailed in Materials and Methods, each recording session contained trials with no stimulus rats present, and with one or two stimulus rats in different locations. For a subset of experiments, inanimate objects were used as stimuli in addition to the stimulus rats.

Spiking activity was recorded from seven rats in a total of 59 recording sessions, yielding a total of 752 neuronal clusters. Cluster separation was quantified using L-ratio and Isolation Distance (Schmitzer-Torbert et al., 2005). Clusters were considered single neurons when they had a clear refractory period. Finally, neurons were classified as putative pyramidal cells if their firing rates were $<5 \mathrm{~Hz}$; otherwise, they were considered interneurons and not further analyzed. The core data consisted of 319 well separated, single, putative pyramidal neurons recorded from six of the seven rats. The main results stayed the same when we considered an extended dataset of less well separated units with a total of 455 neurons (Table 1). The actual number of distinct cells that were sampled is likely lower because recording from close-by locations in consecutive sessions can lead to an overlap in the sampled cells. However, a reanalysis of the data using one single recording per electrode ( $N=113$ well separated neurons) leads to very similar population results ( $\mathrm{Ta}$ ble 1). The true number of distinct neurons is hard to determine and may lie somewhere between 113 and 319. In what follows, all statistics cited in the text were calculated on the standard dataset $(N=319)$.

Approximately one-half of the neurons were from $\mathrm{CA} 1$, and the rest from subfields CA1-3, the DG, and the FC of 
the dorsal hippocampus (Table 2). The FC is the medial extension of CA1, where the pyramidal cell layer curves upward [according to Henriksen et al. (2010), even though denoted CA1 in the rat atlas (Paxinos and Watson, 2007)]. Dorsal CA1 was the main focus of our analysis because previous studies had suggested a prominent role in social recognition and memory (Lai et al., 2005).

In a separate set of immediate-early gene expression experiments, a subject rat was placed in a familiar environment that either contained an unfamiliar rat or no other animals. As in the electrophysiology experiment, snout-tosnout interaction with the other rat was possible across a gap. After the behavioral session, the subject rat's brain was sectioned and stained for c-Fos expression.

\section{Rats are attracted by their conspecifics} In the electrophysiology experiments, the presence of a stimulus rat affected the behavior of the subject rat. Rats would typically lean out toward each other and make contact with their snouts and whiskers. Figure $1 B$ shows how the subject rat was attracted to the gap when there was a stimulus rat present. Moreover, subjects would usually lean out further toward a stimulus rat than toward an empty platform $(p=0.001$, two-tailed permutation test; Fig. 1C).
Table 3. Types of responsive cells

\begin{tabular}{|c|c|c|c|c|}
\hline & \multicolumn{2}{|l|}{ CA1 } & \multicolumn{2}{|c|}{ All recorded regions } \\
\hline & No. & Fraction (\%) & No. & Fraction (\%) \\
\hline Putative single pyramidal cells & 165 & 100 & 319 & 100 \\
\hline Individual-specific cells & 1 & 1 & 3 & 1 \\
\hline Rat-modulated cells & 9 & 5 & 22 & 7 \\
\hline Significant spatial information & 142 & 86 & 274 & 86 \\
\hline Place cells & 104 & 63 & 170 & 53 \\
\hline
\end{tabular}

Significance was calculated at the $1 \%$ level, without correction for multiple testing. A false-positive rate of $\sim 1 \%$ is therefore expected.

\section{Few neurons respond to rats in general, and no neuron responds to individual rats}

The analysis of the subject rat's social behavior could be reduced to a spatial analysis because the stimulus rats were constrained to the small platforms: whenever the subject rat's head was in one of the two gaps in front of a stimulus rat (Fig. $1 \mathrm{~A}$ ), this was considered an interaction. Neuronal firing rates during these times were compared with episodes when the rat was in the same place but there was no stimulus rat present.

This analysis allowed discrimination between the following response profiles: an "individual-specific cell" should fire for one stimulus rat only, in any location (i.e., either one of the two stimulus platforms) where that stimulus rat is placed. A "rat cell" would specifically fire when any stimulus rat is present, regardless of location. A "conjunctive cell” would be modulated in firing by both space and stimulus rat presence. A "place cell" would fire in one or both of the gaps (or have a place field somewhere else), and not be influenced by the presence or identity of the stimulus rats.

In our data, there were conjunctive spatial-socially modulated cells, which were either enhanced or inhibited in their firing rate by the presence of a rat (Fig. 2). While a few cells showed social modulation in both gaps (Fig. $2 A$ ), none of them was convincingly independent of space; for example, the cell in Figure $2 \mathrm{~A}$ showed a reduced response to the gaps even in the absence of the stimulus rats.

In sessions with more than one stimulus rat $(N=267$ of 319 neurons), we determined whether there were individual-specific cells. To this end, we defined an IMI (see Materials and Methods), which quantified the difference in firing rate when the subject rat interacted with one rat as opposed to another. However, neither for single neurons nor at the population level was there a significant effect (population $p=0.11 ; 3$ of 267 single neurons had a $p \leq 0.01$, which is expected by chance; one-tailed permutation tests). Thus, there was no evidence for individual-specific cells in dorsal hippocampus (Table 3).

\section{Conspecifics modulate hippocampal responses}

To quantify the modulation by any stimulus rat, a RMI was defined (see Materials and Methods). It could range from -1 (fired only when no rat is present) to 0 (no modulation) to +1 (fired only when rat is present). It was calculated from firing rates averaged across trials and the two stimulus platforms, for all the times when the subject rat's head was in one of the gaps.

Neurons showed a wide range of RMI values (Fig. $3 A$ ). A total of 22 of 319 pyramidal neurons had an RMI significantly different from chance at the $p=0.01$ level [one-tailed permutation test on abs(RMI); with $N=319$, approximately three false positives are expected]; see Figure 2 for examples of significant cells and Table 3 for a statistical summary.

At the population level, the mean absolute RMI was 0.26 and significantly greater than expected by chance $[p=0.001$, onetailed permutation test on abs(RMI); for CA1 only, $\mathrm{RMI}=0.24$, $p=0.001]$, confirming that the neurons, or at least a subset of them, were indeed modulated in their activity by the presence of stimulus rats.

What characterizes the small subset of rat-responsive cells? Comparing raw firing rates rather than normalized indices (Fig. $3 B$ ) revealed that the significantly modulated neurons $(p<0.01$, shown in black) are thinly scattered across the whole range of firing rates encountered.

A more fine-grained analysis of the behavior compared firing rates during active snout-to-snout interactions to episodes when the subject rat was in the same place without a stimulus rat being present. This was done for a subset of cells $(N=100)$, and the interaction RMI (iRMI) thus obtained was significantly greater than chance [population mean absolute $\mathrm{iRMI}=0.42 ; p=0.01$, 
A

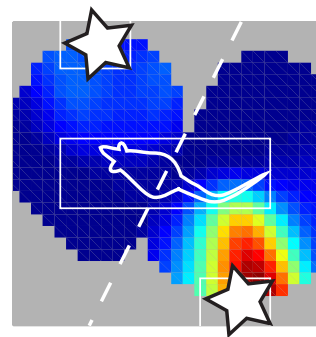

Max Rate 5.2

B

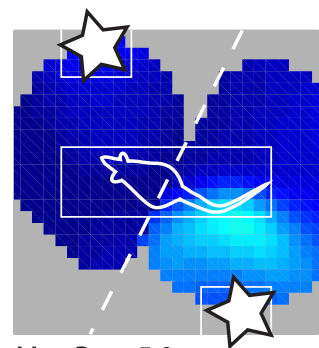

Max Rate 5.9

C
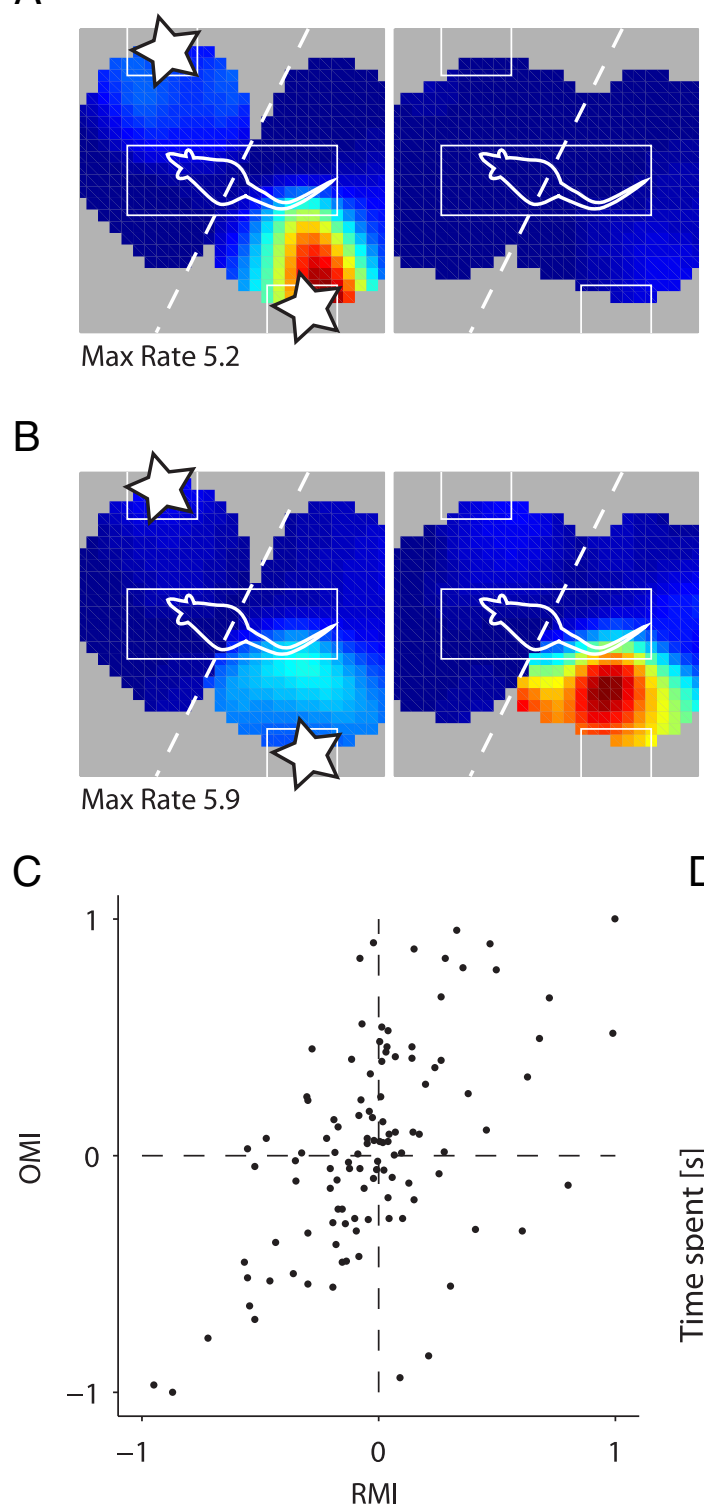
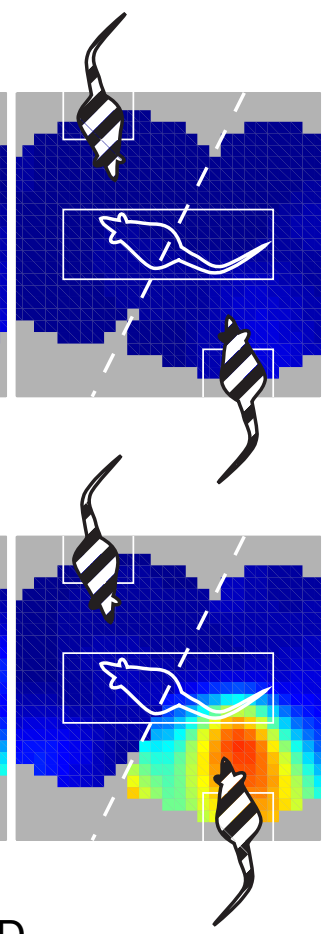

$\mathrm{D}$

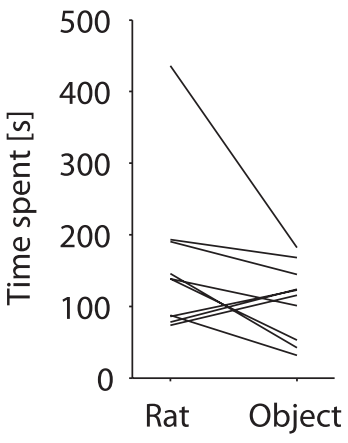

Figure 4. Object-modulated cells. $A$, An example object-modulated cell. Using conventions similar to those of Figure 2, the panels show firing rates averaged across trials with the same stimuli: on the left panel, inanimate objects; middle panel, nothing; right panel, either one of two stimulus rats. As for the rat-modulated cells, object modulation tended to be restricted to one of the gaps. $\boldsymbol{B}, \mathrm{A}$ cell whose firing is suppressed by the object, but not by rats. C, Rat versus object modulation indices of all cells. Note the trend for stronger 0 Mls. D, Each line indicates, for a given recording session, the time the subject rat spent near the stimulus rats and the object.

one-tailed permutation test on abs(iRMI)]. The RMI calculated by spatial analysis (sRMI), as described previously was, for the same selection of neurons, significantly lower $(\mathrm{sRMI}=0.29 ; p=$ 0.001 ). This suggests that it was the social interactions that modulated firing rates more than the mere presence of a conspecific.

Still, there was no sign of neurons discriminating between rats, as the individual modulation index IMI calculated on this subset $(N=$ 78 neurons with multiple rats) remained nonsignificant.

\section{Object interactions affect hippocampal activity more strongly than social interactions}

To assess how responses to social interactions compared to interactions with objects, we let a subset of subject rats (two of seven) interact with rats and inanimate objects. To compare responses, we defined a rat-versus-object index similar to the above RMI, as the difference between firing rates while close to a stimulus rat and close to an object, divided by their sum. Across the 115 neurons recorded in this subset, the mean absolute ratversus-object index (ROI) was 0.34 and significantly different from zero $[p=0.001$, one-tailed permutation test on abs(ROI)]. This means that, on average, neurons did discriminate between rats and objects. The average signed ROI was -0.04 and significantly different from zero $(p<0.05$, twotailed permutation test on signed ROI), indicating that neurons tended to fire slightly less for rats than for objects.

To examine object coding in its own right, an object modulation index (OMI) was defined, analogous to the RMI. The mean absolute OMI was 0.34 and significantly different from zero $[p=0.001$, onetailed permutation test on abs(OMI)]. Examples of object-modulated cells are shown in Figure 4, $A$ and $B$.

Having established that neurons encode rat and object presence, we next asked which of the two was more strongly encoded. The mean absolute RMI and OMI were 0.24 and 0.33 , respectively, for the data set with object interactions, and the OMI was significantly greater ( $p=0.007$, two-tailed permutation test), meaning that neurons are more strongly modulated by inanimate objects than by rats. Figure $4 C$ shows the RMIs and OMIs of all neurons and a trend for OMIs to be stronger is visible.

Was the object modulation stronger than the rat modulation in this subset because of an atypically weak rat effect? This could be ruled out because the mean absolute RMI for with- and without-objects subsets were 0.24 and 0.26 , respectively, and not significantly different $(p=0.4$, two-tailed permutation test).

How does the stronger encoding of objects compare with the subject rats' behavior? To answer this question, we measured how much time the subject rat spent close to the stimulus rat or the object. The analysis was restricted to trials when both stimuli were present to ensure that subject rats had a true choice. Figure $4 D$ shows that, in most sessions, the subject rat spent more time close to the stimulus rat. To quantify the difference, rat and object times were normalized by their sum for a given session. The average normalized times were significantly different $(p=$ 0.037 , two-tailed permutation test). Thus, while rats show greater interest in conspecifics compared with inanimate, inedible objects, responses of hippocampal neurons are more strongly affected by the objects.

\section{Response modulation by rats and objects is weaker than spatial response modulation}

Spatially modulated cells were detected by two independent criteria. First, place fields were identified as contiguous areas of firing activity (Muller et al., 1987) (see Materials and Methods). 
Consistency was assessed by calculating Pearson's correlation coefficient between the firing maps of random temporal subsets of a recording session. To be considered a place cell, a neuron had to have at least one place field, and its consistency was required to be $>50 \%$. A total of 170 of 319 neurons, 53\%, satisfied these criteria (Table 3), a fraction slightly higher than previous reports (Wilson and McNaughton, 1993). It is known that salient stimuli like objects lead to the expression of more place fields (Burke et al., 2011), and this may explain the higher incidence in the present paradigm compared with previous place field studies. As a second alternative measure, the spatial information rate of the neurons was calculated. Significance was estimated by a permutation test that calculated the spatial information on spike data that was time-shifted with respect to the rat's positions (Skaggs et al., 1993) (see Materials and Methods). A total of 274 of the 319 neurons (Table 3 ) was significantly spatially modulated at the $p=0.01$ level (one-tailed permutation test; approximately three false positives are expected), a fraction similar to previous studies using the same criteria (Henriksen et al., 2010). Examples of spatially modulated neurons whose firing was not modulated by the presence of conspecifics are shown in Figure 5.

Thus, while a majority of neurons showed spatial coding, only a few neurons were modulated by rats or objects. This raises the question to what extent these populations overlapped; i.e., were the rat and place responses place independent or did cells respond to a combination of place and rat (or object) presence?

As discussed above, there are no truly convincing examples of place-independent rat cells. To examine this at the population level, however, a PMI was defined, designed to be analogous in definition to the RMI. Briefly, PMI was the normalized difference in firing rate between the two gap zones, averaging across all trials with and without stimulus rats. Across all neurons, the average absolute value of PMI was 0.48 [significantly greater than chance, $p=0.001$, one-tailed permutation test on abs(PMI) $]$. A total of 140 of 319 neurons had significant place modulation across the two gaps at the $p=0.01$ level.

Relating the PMIs of the cells to their RMIs showed that ratmodulated cells (Fig. 5C, dots in the top part) had varying degrees of spatial modulation. PMI and RMI were not related in any obvious way, and Pearson's correlation coefficient $c$ was not significant ( $c=-0.018, p=0.75$, two-tailed $t$ test). In an analogous way, place coding and object coding were uncorrelated across cells (Fig. 5D; $c=-0.035, p=0.71$ ). As a consequence, one is left with the null hypothesis that coding for place on the one hand and rat or object presence on the other hand are independent properties. While this predicts that, statistically, one should ob- serve a fraction of cells that encode rats or objects independently of space, there were no fully convincing examples in our data set.

\section{Social encounters induce c-Fos expression in the amygdala but not in the hippocampus}

The rat hippocampus is a large and regionally heterogeneous brain structure. The absence of individually specific responses and the low incidence of socially modulated neurons in our data set could thus easily result from incomplete sampling (i.e., be a false-negative result). To address this concern, we performed immunohistochemistry of socially induced c-Fos expression. This method allows visualization of immediate-early gene activity in all of the neurons of the brain. The results from the early gene expression experiments were consistent with the conclusions from the electrophysiological experiments: hippocampus, in particular dorsal CA1, showed very low densities of c-Fos-positive cells (Fig. 6, Table 4) in both conditions (social interaction and control). To estimate the fraction of cells that were $\mathrm{c}$-Fos positive, a total cell count was performed for dorsal CA1 on the Nisslstained sections. The cell density was 333,276 \pm 60,780 cells/ 



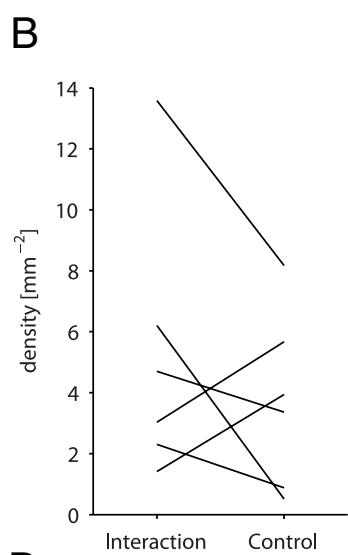

D
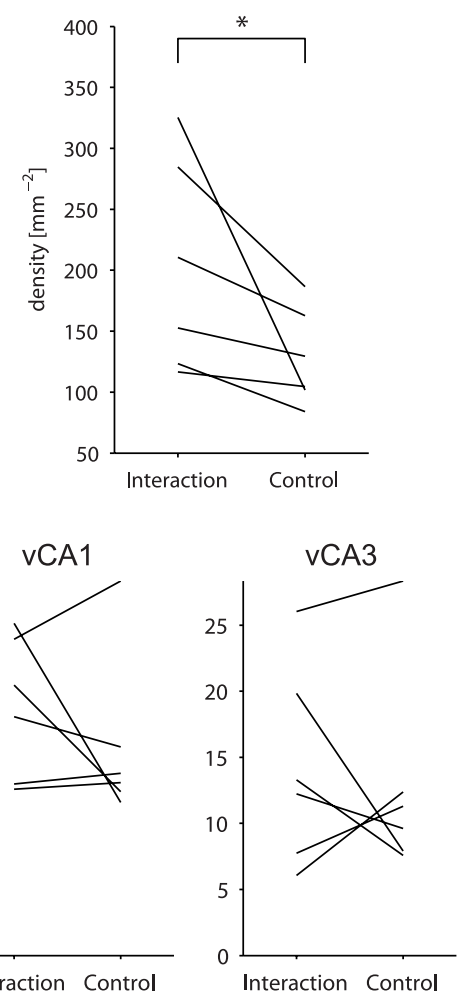

Figure 6. c-Fos activation across regions. $A, C$-Fos staining in the CA1 subfield of the dorsal hippocampus. The red outline in the Niss-stained section (left) indicates the area shown in the $c$-Fos stains (center and right, social interaction and control condition, respectively). Only very few cells were c-Fos positive (see zoomed area). B, Average density of c-Fos-positive cells per animal. The lines connect pairs of animals that underwent immunohistochemical processing jointly. There was no significant difference between the social interaction and control condition. $C, D, S a m e$ as $\boldsymbol{A}$ and $\boldsymbol{B}$ for the basolateral amygdala. There was a significant increase with social interaction, as indicated by the asterisk. E, c-Fos cell densities for other hippocampal subfields. None had a significant change with social interaction.

Table 4. Summary of c-Fos results

\begin{tabular}{|c|c|c|c|c|c|}
\hline \multirow[b]{3}{*}{ Region } & \multicolumn{4}{|c|}{ Cell density $\left(n / \mathrm{mm}^{2}\right)$} & \multirow{3}{*}{$\begin{array}{l}p \text { value of } \\
\text { difference }\end{array}$} \\
\hline & \multicolumn{2}{|c|}{ Social interaction } & \multicolumn{2}{|c|}{ Control } & \\
\hline & Mean & SD & Mean & SD & \\
\hline dCA1 & 5.2 & 4.5 & 3.8 & 2.9 & 0.28 \\
\hline $\mathrm{dCA} 2$ & 24.5 & 20.3 & 22.8 & 14.1 & 0.52 \\
\hline $\mathrm{dCA} 3$ & 9.1 & 3.2 & 10.5 & 5.2 & 0.61 \\
\hline dDG & 9.8 & 2.5 & 13.6 & 8.0 & 0.77 \\
\hline $\mathrm{dFC}$ & 3.6 & 3.7 & 2.4 & 1.6 & 0.28 \\
\hline vCA1 & 32.2 & 9.1 & 27.0 & 10.7 & 0.19 \\
\hline vCA3 & 14.2 & 7.5 & 12.9 & 7.8 & 0.36 \\
\hline BLA & 202 & 87 & 128 & 40 & 0.02 \\
\hline
\end{tabular}

For each brain region considered, means and SDs of the treatment and control groups are given $(N=6$ animals each). SDs were calculated across animals, not within animals. See Materials and Methods for statistics details. $\mathrm{mm}^{3}$ (mean $\pm \mathrm{SD}$ ). This means that as few as $0.04 \%$ of CA1 cells were c-Fos positive in the social interaction condition.

This was in contrast with high levels of c-Fos expression in other brain regions like the cortex (not quantified but visible in Fig. $6 A$ ) and the BLA (Fig. 6C,D, Table 4). Moreover, there was no main effect of treatment on c-Fos expression; none of the dorsal hippocampal areas recorded from (CA1, CA2, CA3, DG, and FC) showed a significant difference in expression between rats that had social interactions and those that did not (Fig. 6E, Table 4). This is unlikely a failure of the behavioral paradigm to elicit differential brain activation because there was a significant effect ( $p<0.05$, one-tailed permutation test) of social interaction in the basolateral amygdala (Fig. 6C,D).

Unlike the dorsal hippocampus, the ventral hippocampus has strong connectivity with regions such as the amygdala and the 
hypothalamus, which has led to the hypothesis that it is involved in emotional processing. Moreover, it receives strong olfactory input through entorhinal cortex (Fanselow and Dong, 2010). Together, this may make it appear as a more likely substrate of social recognition and memories than dorsal hippocampus. However, our c-Fos data show low activity and no social interactioninduced enhancement in ventral CA1 and CA3 (Fig. 6E, rightmost panels; Table 4).

The interpretation of this null result is ambiguous because there is no universal consensus regarding the meaning of c-Fos staining; it is at times considered a sign of previous action potential activity (Barth et al., 2004), and other times a trace of neuronal plasticity (Ito and Schuman, 2011). In the present context, these two interpretations lead to different predictions: if ventral hippocampus did, indeed, encode social stimuli, and if it did so by making some neurons fire less and some more, as observed in the neurons recorded in dorsal hippocampus, then the net effect of social interaction with novel individuals might be unaltered bulk activity, but the formation of new memories should lead to increased plasticity. Therefore, if c-Fos reflects plasticity, then social interaction should increase c-Fos staining, but not necessarily if it reflects activity.

\section{Rat responses are heterogeneous within CA1}

The recording sites within CA1 differed widely between electrodes and subject rats. While the heterogeneous responses observed at different locations sample CA1 too sparsely to prove a pattern, anatomy suggests one: Direct inputs from the entorhinal cortex to CA1 are structured along the proximodistal axis (Steward, 1976; Amaral and Witter, 1995). Moreover, spatial and nonspatial processing are thought to be localized in the medial and lateral entorhinal cortex, respectively (Hargreaves et al., 2005; Knierim et al., 2006; Manns and Eichenbaum, 2006). Therefore, nonspatial signals could be expected to be stronger in distal CA1 compared with proximal CA1, and this may hold for social representations, too. The present data are consistent with this prediction: Comparison of the proximodistal coordinates with the respective rat modulation indices reveals a clear trend for more distal neurons to show a wider range of RMIs. The correlation across cells between the proximodistal coordinate and the absolute RMI is significant (Pearson's correlation coefficient $c=0.22$, $p<0.01$, two-tailed $t$ test).

Conversely, spatial coding has been reported to be degraded at more distal positions (McNaughton et al., 2008; Henriksen et al., 2010). Comparing the spatial information of neurons with their proximodistal score reveals a significant negative correlation $(c=$ $-0.20, p<0.01$, two-tailed $t$ test), indicating that more distal neurons carry lower spatial information, as reported in the above-mentioned studies.

If social responses were, indeed, stronger at more distal positions within CA1, then this should be visible in the early gene expression data. To test this, a distal score ( 0 near CA2, 1 near the FC) was calculated for all c-Fos-positive cells. However, the distal score averaged across cells of each rat was not significantly greater for rats that had had social interactions compared with control ( $p=0.21$, permutation test, one-tailed). Nor was there a bulk increase in c-Fos activation with social interaction, as noted in the previous section (Fig. $6 A, B$ ).

Thus, while the physiology data are clearly suggestive of a regional differentiation along the known anatomical gradient, this is not obvious from the c-Fos data.

\section{Discussion}

The present study examined, in separate electrophysiology and c-Fos expression experiments, neuronal activation in response to social interaction with conspecifics.

We recorded putative pyramidal neurons from the CA1, CA2, CA3, DG, and FC subfields of the dorsal rat hippocampus and asked whether there were cells responsive to conspecifics in general (rat cells), or even to individual rats (individual-specific cells), and whether these responses were linked to the well described spatial responses of the rodent hippocampus. We found no individual-specific cells, but we observed that some neurons reacted to the presence of stimulus rats with an enhancement or a suppression of their firing rate. These rat-modulated cells showed conjunctive spatial-social responses and were spatially modulated to varying degrees.

The c-Fos experiments were designed to gain an overview of the brain areas, within and beyond the hippocampus, that are implicated when rats interact socially. We found very few c-Fospositive neurons in the dorsal and the ventral hippocampus, particularly in CA1, and their number was not greater in rats that had social interactions compared with those that did not. Together, the physiology and the c-Fos data suggest that social representations do not play a prominent role in the rat hippocampus.

\section{Absence of individual-specific cells in dorsal hippocampus}

Among 267 neurons from sessions with multiple stimulus rats, there were no significantly individual-specific cell, after correcting for multiple testing. The rat-modulated cells that were observed showed conjunctive responses to stimulus rats and place. This indicates that, in the present paradigm, neurons in the dorsal hippocampus were not strongly concerned with social stimuli, but mainly showed the well characterized spatial memory (O’Keefe and Nadel, 1978; Muller, 1996).

Our recordings did not uniformly sample CA1, and even less so the other subfields, meaning that a hypothetical small, socially responsive subregion might have gone unnoticed. Our c-Fos experiments did not reveal any such region, though. While there was a significant increase in c-Fos expression for socially interacting rats in the basolateral amygdala, no such differential activation was observed in the hippocampus, in both its ventral and dorsal part. However, as pointed out in Results, there is the possibility that encoding of social stimuli was present but not reflected by c-Fos staining. Therefore, the lack of staining in ventral hippocampus argues against but does not entirely rule out its involvement in social recognition.

A possible explanation for the lack of individual discrimination by the recorded neurons is the hypothesis that subject rats did not actually individually recognize the stimulus rats. Given the highly developed individual recognition abilities of rats and other rodents, we think this is unlikely.

A weaker form of the above is the idea that the subject rats did distinguish between the stimulus rats, but due to the low behavioral relevance of the discrimination in the given setting, hippocampal neurons did not encode the difference. Indeed, a study addressing discrimination among inanimate objects found that, in a task-free paradigm, neurons clearly signaled the objects' presence and their location, but carried much less information about their identity (Manns and Eichenbaum, 2009).

The fact that cells were modulated by a combination of place and rat presence, but not by rat identity, calls into question their social specificity. Does the neuronal population recorded from encode conspecifics in terms of their social value or merely as 
obstacles in space, as described previously for inanimate objects (Muller and Kubie, 1987; Rivard et al., 2004)?

The present experiments with objects showed that neurons do discriminate between rats and objects. Therefore, even though the neuronal responses reported here lack individual specificity, they are specifically social in that they make a difference between rats and objects. Surprisingly, they were more strongly modulated by objects than by stimulus rats, even though the subject rats spent more time interacting with their conspecifics than with the objects. This clearly rules out a privileged role for social signals in at least the dorsal part of the rodent hippocampus.

The absence of individual-specific signals in pyramidal cells of dorsal CA1 is in contrast with reports of human hippocampal cells that responded selectively to representations of individual persons (Quian Quiroga et al., 2005, 2008, 2009). Cells shown in these studies had remarkable invariance to stimulus features: one neuron responded to each one of a set of different photographs of the actress Jennifer Aniston, while showing no response at all to images of, for example, other blond women. The cell even fired for her name, both in writing and as spoken word.

Another suggestive example of neurons with abstract, featureinvariant response properties was the report of "nest cells," hippocampal neurons that fired for a mouse's nest whenever the animal was in it, no matter where the nest was located (Lin et al., 2007).

Inspired by these and other studies, we were looking for a single-neuron, firing rate code of conspecifics. While in this framework we did not find individual-specific coding, the existence of, for example, a spike timing or multineuron code cannot be ruled out.

It is unclear how the high specificity of hippocampal responses in these studies can be reconciled with the unspecific responses in the present data or in the study by Manns and Eichenbaum (2009); we can only speculate that the salience and relevance of a stimulus plays a crucial role.

What could be the function of the observed socially modulated cells? Some authors suggest that in rodents, like in primates, the hippocampus processes episodic memories (Simpson et al., 1988; Gaffan et al., 2001; Eacott and Norman, 2004). In this perspective, conjunctive cells might be part of an episodic memory trace, linking the stimulus rat to the place where it was observed. Consistent with this view, experiments with hamsters showed that, when dorsal CA1 was reversibly inactivated during an encounter with familiar individuals, the subject failed to associate the respective memories (Lai et al., 2005).

On the other hand, most lesion studies (Bannerman et al., 2001; Petrulis and Eichenbaum, 2003; Squires et al., 2006; Feinberg et al., 2012) showed that rodents do not require an intact hippocampus to recognize conspecifics as familiar. Our data confirm and extend these findings by demonstrating a lacking individual specificity of neural signals and an absence of immediate-early gene expression after social encounters.

\section{Spatial, nonspatial, and social responses}

While we know of no previous study that measured hippocampal responses to other rats from a social coding perspective, there have been reports of modified place field firing in the presence of other rats (Zynyuk et al., 2011). Mainly, the spatial coherence and firing rates of place fields were reduced by conspecifics, and more so the closer the other rat was. While Zynyuk et al. (2011) did not examine individual-specific responses, their conclusions agree with the present study in the sense that, overall, the impact of social stimuli on pyramidal cell firing in the dorsal hippocampus is weak.

In addition to the extensive literature on spatial representations in the rodent hippocampus, responses to nonspatial stimuli have been described as well. While animals were performing olfactory (Eichenbaum et al., 1987; Otto and Eichenbaum, 1992; Wood et al., 1999; Wiebe and Stäubli, 2001; Deshmukh and Bhalla, 2003; Komorowski et al., 2009), auditory (Sakurai, 1994; Moita et al., 2003), visual (Wible et al., 1986), tactile (Pereira et al., 2007; Itskov et al., 2011), and spatial (Deadwyler et al., 1996) tasks, hippocampal neurons encoded task-related stimuli, the time course of the task, error signals, space, or conjunctions thereof.

In the present study, neuronal responses to social stimuli were weaker than to objects, and both were much less prominent than spatial representations. While some previous studies have reported the relative prevalence of spatial and nonspatial responses as comparable (Wible et al., 1986; Young et al., 1994; Wood et al., 1999), others found that spatial responses dominated (Komorowski et al., 2009; Manns and Eichenbaum, 2009). Among the reasons for the low incidence of rat-responsive cells in the present study may be the lack of a task involving the stimulus rats. Indeed, the share of neurons that encode nonspatial items has been shown to increase dramatically when they are linked to a task (Moita et al., 2003; Komorowski et al., 2009).

Modulation of place field firing has also been described in the framework of remapping, whereby some or all place cells change their firing fields (partial and global remapping, respectively) or undergo changes in firing rate while maintaining the locations of their place fields (rate remapping). While global remapping occurs between different global environment (Leutgeb et al., 2005), changes in the local environment, task conditions, or cognitive state lead to partial or rate remapping (Leutgeb et al., 2005; Jackson and Redish, 2007). For the present paradigm, partial or rate remapping would be expected because, except for the stimulus rats, the environment was static. Indeed, each rat-modulated cell reported here can be interpreted as being part of distinct maps, differentiated by the in-field firing rates of the cell, which are activated in the presence and absence of stimulus rats. Consistent with this, a previous study looking at place field modulations in the presence of another rat (Zynyuk et al., 2011) found no global and only modest rate remapping. Our data were not analyzed in a remapping perspective, though, because we considered our analysis more straightforward for the social coding-related questions we asked.

\section{Heterogeneity of social responses within CA1}

CA1 receives structured input through the temporoammonic pathway: lateral and medial entorhinal cortex project to the distal and proximal parts, respectively, of CA1 (Steward, 1976; Amaral and Witter, 1995). Recently, place field properties have been reported to vary along the proximodistal axis (McNaughton et al., 2008; Henriksen et al., 2010), and proximal neurons are more strongly spatially modulated (Henriksen et al., 2010). Conversely, c-Fos studies have shown selective distal activation in response to nonspatial stimuli in mice and rats (Ito et al., 2010; Ito and Schuman, 2011).

The present study showed striking differences in social responses as a function of recording location. We therefore examined whether these differences were structured along the proximodistal axis, like in the studies mentioned above. The physiology data did, indeed, show stronger social signals in the distal than in the proximal part of CA1, and the reverse gradient was observed for spatial signals. The c-Fos 
data, however, showed no such contrast. Given that early gene expression staining is a very sensitive technique for the detection of regional differences, the joint data do not indicate an anatomical structure of social responses.

\section{Conclusion}

The present study examined social coding in the rat brain while interacting with conspecifics. The social stimulus responses were less prominent than, and partly embedded in, the well known spatial memory of the hippocampus. Together with the lack of activation of hippocampus by social interactions in the c-Fos paradigm, these data speak against social representations as a primary function of dorsal hippocampal cells in the rat.

\section{References}

Amaral D, Witter MP (1995) Hippocampal formation. In: The rat nervous system (Paxinos G, ed), pp 443-494. San Diego: Academic.

Bannerman DM, Lemaire M, Beggs S, Rawlins JN, Iversen SD (2001) Cytotoxic lesions of the hippocampus increase social investigation but do not impair social-recognition memory. Exp Brain Res 138:100-109.

Barth AL, Gerkin RC, Dean KL (2004) Alteration of neuronal firing properties after in vivo experience in a FosGFP transgenic mouse. J Neurosci 24:6466-6475.

Burke SN, Maurer AP, Nematollahi S, Uprety AR, Wallace JL, Barnes CA (2011) The influence of objects on place field expression and size in distal hippocampal CA1. Hippocampus 21:783-801.

Burwell RD (2000) The parahippocampal region: corticocortical connectivity. Ann N Y Acad Sci 911:25-42.

Deadwyler SA, Bunn T, Hampson RE (1996) Hippocampal ensemble activity during spatial delayed-nonmatch-to-sample performance in rats. J Neurosci 16:354-372.

Deshmukh SS, Bhalla US (2003) Representation of odor habituation and timing in the hippocampus. J Neurosci 23:1903-1915.

Eacott MJ, Norman G (2004) Integrated memory for object, place, and context in rats: a possible model of episodic-like memory? J Neurosci 24:1948-1953.

Eichenbaum H, Kuperstein M, Fagan A, Nagode J (1987) Cue-sampling and goal-approach correlates of hippocampal unit activity in rats performing an odor-discrimination task. J Neurosci 7:716-732.

Eichenbaum H, Dudchenko P, Wood E, Shapiro M, Tanila H (1999) The hippocampus, memory, and place cells: is it spatial memory or a memory space? Neuron 23:209-226.

Fanselow MS, Dong HW (2010) Are the dorsal and ventral hippocampus functionally distinct structures? Neuron 65:7-19.

Feinberg LM, Allen TA, Ly D, Fortin NJ (2012) Recognition memory for social and non-social odors: differential effects of neurotoxic lesions to the hippocampus and perirhinal cortex. Neurobiol Learn Mem 97:7-16.

Gaffan EA, Bannerman DM, Warburton EC, Aggleton JP (2001) Rats' processing of visual scenes: effects of lesions to fornix, anterior thalamus, mamillary nuclei or the retrohippocampal region. Behav Brain Res 121:103-117.

Gheusi G, Goodall G, Dantzer R (1997) Individually distinctive odours represent individual conspecifics in rats. Anim Behav 53:935-944.

Hargreaves EL, Rao G, Lee I, Knierim JJ (2005) Major dissociation between medial and lateral entorhinal input to dorsal hippocampus. Science 308:1792-1794

Harris KD, Hirase H, Leinekugel X, Henze DA, Buzsáki G (2001) Temporal interaction between single spikes and complex spike bursts in hippocampal pyramidal cells. Neuron 32:141-149.

Henriksen EJ, Colgin LL, Barnes CA, Witter MP, Moser MB, Moser EI (2010) Spatial representation along the proximodistal axis of CA1. Neuron $68: 127-137$.

Husted JR, McKenna FS (1966) The use of rats as discriminative stimuli. J Exp Anal Behav 9:677-679.

Ito HT, Schuman EM (2011) Functional division of hippocampal area CAl via modulatory gating of entorhinal cortical inputs. Hippocampus. Advance online publication. Retrieved January 10, 2012. doi:10.1002/hipo.20909.

Ito HT, Smith SE, Hsiao E, Patterson PH (2010) Maternal immune activation alters nonspatial information processing in the hippocampus of the adult offspring. Brain Behav Immun 24:930-941.
Itskov PM, Vinnik E, Diamond ME (2011) Hippocampal representation of touch-guided behavior in rats: persistent and independent traces of stimulus and reward location. PLoS One 6:e16462.

Jackson J, Redish AD (2007) Network dynamics of hippocampal cell assemblies resemble multiple spatial maps within single tasks. Hippocampus 17:1209-1229.

Johnston RE, Jernigan P (1994) Golden hamsters recognize individuals, not just individual scents. Anim Behav 48:129-136.

Knierim JJ, Lee I, Hargreaves EL (2006) Hippocampal place cells: parallel input streams, subregional processing, and implications for episodic memory. Hippocampus 16:755-764.

Kogan JH, Frankland PW, Silva AJ (2000) Long-term memory underlying hippocampus-dependent social recognition in mice. Hippocampus 10:47-56.

Komorowski RW, Manns JR, Eichenbaum H (2009) Robust conjunctive item-place coding by hippocampal neurons parallels learning what happens where. J Neurosci 29:9918-9929.

Lai WS, Ramiro LL, Yu HA, Johnston RE (2005) Recognition of familiar individuals in golden hamsters: a new method and functional neuroanatomy. J Neurosci 25:11239-11247.

Leutgeb S, Leutgeb JK, Barnes CA, Moser EI, McNaughton BL, Moser MB (2005) Independent codes for spatial and episodic memory in hippocampal neuronal ensembles. Science 309:619-623.

Lin L, Chen G, Kuang H, Wang D, Tsien JZ (2007) Neural encoding of the concept of nest in the mouse brain. Proc Natl Acad Sci USA 104:6066-6071.

Manns JR, Eichenbaum H (2006) Evolution of declarative memory. Hippocampus 16:795-808.

Manns JR, Eichenbaum H (2009) A cognitive map for object memory in the hippocampus. Learn Mem 16:616-624.

McNaughton BL, Hoang LT, Valdes JL, Maurer AP, Burke SN, Fellous J-M (2008) Distinct characteristics of CA1 place cells correlated with medial or lateral entorhinal cortex layer III input. Soc Neurosci Abstr 34:391.3.

Moita MA, Rosis S, Zhou Y, LeDoux JE, Blair HT (2003) Hippocampal place cells acquire location-specific responses to the conditioned stimulus during auditory fear conditioning. Neuron 37:485-497.

Montemurro MA, Senatore R, Panzeri S (2007) Tight data-robust bounds to mutual information combining shuffling and model selection techniques. Neural Comput 19:2913-2957.

Muller R (1996) A quarter of a century of place cells. Neuron 17:813-822.

Muller RU, Kubie JL (1987) The effects of changes in the environment on the spatial firing of hippocampal complex-spike cells. J Neurosci 7:1951-1968

Muller RU, Kubie JL, Ranck JB Jr (1987) Spatial firing patterns of hippocampal complex-spike cells in a fixed environment. J Neurosci 7:1935-1950.

O’Keefe J (1999) Do hippocampal pyramidal cells signal non-spatial as well as spatial information? Hippocampus 9:352-364.

O'Keefe J, Nadel L (1978) The hippocampus as a cognitive map. Oxford: Oxford UP.

Otto T, Eichenbaum H (1992) Neuronal activity in the hippocampus during delayed non-match to sample performance in rats: evidence for hippocampal processing in recognition memory. Hippocampus 2:323-334.

Paxinos G, Watson C (2007) The rat brain in stereotaxic coordinates. San Diego: Academic.

Pereira A, Ribeiro S, Wiest M, Moore LC, Pantoja J, Lin SC, Nicolelis MA (2007) Processing of tactile information by the hippocampus. Proc Natl Acad Sci U S A 104:18286-18291.

Petrulis A (2009) Neural mechanisms of individual and sexual recognition in Syrian hamsters (Mesocricetus auratus). Behav Brain Res 200:260-267.

Petrulis A, Eichenbaum H (2003) The perirhinal-entorhinal cortex, but not the hippocampus, is critical for expression of individual recognition in the context of the Coolidge effect. Neuroscience 122:599-607.

Quian Quiroga R, Reddy L, Kreiman G, Koch C, Fried I (2005) Invariant visual representation by single neurons in the human brain. Nature 435:1102-1107.

Quian Quiroga R, Kreiman G, Koch C, Fried I (2008) Sparse but not "Grandmother-cell" coding in the medial temporal lobe. Trends Cogn Sci 12:87-91.

Quian Quiroga R, Kraskov A, Koch C, Fried I (2009) Explicit encoding of multimodal percepts by single neurons in the human brain. Curr Biol 19:1308-1313. 
Redish AD (2001) The hippocampal debate: are we asking the right questions? Behav Brain Res 127:81-98.

Rivard B, Li Y, Lenck-Santini PP, Poucet B, Muller RU (2004) Representation of objects in space by two classes of hippocampal pyramidal cells. J Gen Physiol 124:9-25.

Sakurai Y (1994) Involvement of auditory cortical and hippocampal neurons in auditory working memory and reference memory in the rat. J Neurosci 14:2606-2623.

Schmitzer-Torbert N, Jackson J, Henze D, Harris K, Redish AD (2005) Quantitative measures of cluster quality for use in extracellular recordings. Neuroscience 131:1-11.

Scoville WB, Milner B (1957) Loss of recent memory after bilateral hippocampal lesions. J Neurol Neurosurg Psychiatry 10:11-21.

Simpson EL, Gaffan EA, Eacott MJ (1988) Rats' object-in-place encoding and the effect of fornix transection. Psychobiology 26:190-204.

Skaggs W, Mcnaughton B, Gothard K (1993) An information-theoretic approach to deciphering the hippocampal code. In: Advances in neural information processing systems, pp 1030-1037. San Mateo, CA: Morgan Kaufmann.

Squire LR (1992) Memory and the hippocampus: a synthesis from findings with rats, monkeys, and humans. Psychol Rev 99:195-231.

Squires AS, Peddle R, Milway SJ, Harley CW (2006) Cytotoxic lesions of the hippocampus do not impair social recognition memory in socially housed rats. Neurobiol Learn Mem 85:95-101.

Steward O (1976) Topographic organization of the projections from the entorhinal area to the hippocampal formation of the rat. J Comp Neurol $167: 285-314$
Thor DH, Holloway WR (1982) Social memory of the male laboratory rat. J Comp Physiol Psychol 96:1000-1006.

Uekita T, Okanoya K (2011) Hippocampus lesions induced deficits in social and spatial recognition in Octodon degus. Behav Brain Res 219:302-309.

Wible CG, Findling RL, Shapiro M, Lang EJ, Crane S, Olton DS (1986) Mnemonic correlates of unit activity in the hippocampus. Brain Res 399:97-110.

Wiebe SP, Stäubli UV (2001) Recognition memory correlates of hippocampal theta cells. J Neurosci 21:3955-3967.

Wilson MA, McNaughton BL (1993) Dynamics of the hippocampal ensemble code for space. Science 261:1055-1058.

Winocur G (1990) Anterograde and retrograde amnesia in rats with dorsal hippocampal or dorsomedial thalamic lesions. Behav Brain Res 38:145-154.

Wood ER, Dudchenko PA, Eichenbaum H (1999) The global record of memory in hippocampal neuronal activity. Nature 397:613-616.

Young BJ, Fox GD, Eichenbaum H (1994) Correlates of hippocampal complex-spike cell activity in rats performing a nonspatial radial maze task. J Neurosci 14:6553-6563.

Zola-Morgan S, Squire LR, Amaral DG (1986) Human amnesia and the medial temporal region: enduring memory impairment following a bilateral lesion limited to field CA1 of the hippocampus. J Neurosci 6:2950-2967.

Zynyuk L, Huxter J, Muller RU, Fox SE (2011) The presence of a second rat has only subtle effects on the location specific firing of hippocampal place cells. Hippocampus. Advance online publication. Retrieved January 10, 2012. doi:10.1002/hipo.20977. 